\title{
Single-domain antibody based thermally stable electrochemical immunosensor
}

Aparajita Singh, Syed Khalid Pasha, Pandiaraj Manickam, and Shekhar Bhansali*

BioMEMS and Microsystems Laboratory, Department of Electrical and Computer Engineering,

Florida International University, Miami, Florida-33174

*Corresponding author email: sbhansa@fiu.edu, Ph: +1-305-348-2807

\begin{abstract}
Conventional monoclonal and polyclonal antibodies are sensitive to changes in environmental factors such as temperature, $\mathrm{pH}$, humidity, etc. This limits the current costeffective and portable electrochemical immunosensors in harsh environments. Using Ricin Chain-A, a naturally occurring toxin, as a model analyte we report fabrication of a thermally stable electrochemical immunosensor. Single-domain antibodies (sdAb) or nanobodies have been employed as recognition elements for direct detection of Ricin at temperatures great than $4{ }^{\circ} \mathrm{C}$. Immunosensor fabricated using the conventional Ricin monoclonal and polyclonal antibodies have also been demonstrated for comparison. In the case of $\mathrm{sdAb}$ immunosensor, Ricin was detected in a linear range of $1 \log (\mathrm{fg} / \mathrm{mL})-1 \log (\mu \mathrm{g} / \mathrm{mL})$ with a sensitivity of 0.07 $\mu \mathrm{A} / \log (\mathrm{g} / \mathrm{mL}) / \mathrm{cm}^{2}$ using cyclic voltammetry. The fabricated miniaturized sensors have demonstrated higher shelf life and stability at temperatures up to $40{ }^{\circ} \mathrm{C}$. Therefore these electrochemical sensors can be integrated as a part of a portable device for point-of-care immunosensing.
\end{abstract}

Keywords: Electrochemical sensor; Immunosensor; Single-domain antibody; Ricin; Thermalstability 


\section{Introduction}

Immunosensors are affinity-based biosensors that detect the binding of target analytes (antigens) to its specific antibody by their immunochemical reaction at the transducer surface. Over the years, electrochemical sensing has emerged as a highly promising technique for the measurement of clinically relevant analytes. It has satisfied the need of sensing platforms that can be deployed at point-of-care (POC) in order to quantify analytes rapidly in relevant biofluids. It is based on the principle of measuring the changes in electrical properties of a conductive material due to the adsorption of an analyte on the surface functionalized with antibodies (Kaushik et al. 2013; Wan et al. 2013). The electrical change is attributed to the change in the concentration of the electro active redox species at the electrode. With each successive addition of crosslinking layer, immobilization of the antibody, and antigen binding in the sensor matrix, the redox current magnitude decreases. This is because the successive layers are non-conductive and hinder electron transport in the solution. The mature processing capability of the microfabricate industry has allowed the building of microelectrodes that are highly sensitive and provide very low detection limits. The simplicity of electronic circuitry for electrochemical detection and cheap volume manufacturing has driven efforts to bring electrochemical immunosensing up to speed with other immunosensing techniques (Lillehoj et al. 2013; Loncaric et al. 2012). Though miniaturized electrochemical immunosensors have addressed the issues of time-consuming processing, large sample volume requirement, and high cost related to conventional lab based diagnostic methods, there are still challenges that need to be resolved.

The inherent drawback that limits the application of electrochemical based immunosensors in the field is its low shelf life. The sensitivity of the antibodies to the environmental factors such as fluctuation in temperature, change in $\mathrm{pH}$, exposure to light, etc limits the storage capacity. At temperatures higher than the typical storage conditions (usually 4 ${ }^{\circ} \mathrm{C}$ ), the conventional (monoclonal and polyclonal) antibodies suffer from destabilization (denaturation) and lose their ability to bind to specific targets. Attempts have been made to preserve the integrity of proteins by encapsulation using sol-gel chemistry. In this method, the biomolecules to be encapsulated are mixed with silica sol (aqueous alkali metal silicate solution) to form a thin film or a gel (Brinker et al. 2002). This technique has been shown to work in drug delivery systems and biosensors (Brinker et al. 2002; Lan et al. 2000; MacCraith et al. 1995; Swed et al. 2014). However, some underlining issues still need to be investigated prior to using 
the sol-gel material successfully for biosensors. The main concerns include: even distribution of biomolecules within the sol-gel matrix, long term stability and storage, response time of the sensor, and interactions between the sol-gel material, biomolecules, and analyte. To avoid the complex procedure of encapsulation and related issues, we propose a different path to enhance the temperature stability and shelf life of the electrochemical based sensors.

Replacement of these standard antibodies with a type of recognition element that is more stable could enhance the performance of the device and simplify the problem of storage and testing in harsh environments. Aptamers (Radi 2011), conventional antibody fragments (Wang and Duan 2011), and single-domain antibodies (Goldman et al. 2006) have gained recognition as unconventional recognition elements for their high stability over repeated cycles of denaturation and renaturation. They have the advantages of protein based polyclonal and monoclonal antibodies. They are readily accessible by targets. However, they are not available for a wide variety of target analytes. In this paper, a thermally stable immunosensor platform is presented using Ricin Chain-A as the model analyte and single-domain antibody ( $\mathrm{sdAb})$ as the antigen binding molecule. Though the benefits of the sdAb based immunosensing are many, only a little progress has been made in this direction. Some $\mathrm{sdAb}$ based immunosensors that can be found in literature are: amperometric magnetoimmunosensors (Campuzano et al. 2014), electrochemical impedance immunosensors (Zhu et al. 2015), resistive immunosensors (Slocik et al. 2010), and surface plasmon resonance immunosensor (Huang et al. 2005). Successful utilization of specific sdAbs has been demonstrated in detecting various pathogens and toxins (Anderson et al. 2010; Anderson et al. 2008). Yet, the same stability and affinity of these sdAbs is not confirmed for electrochemical platforms. In this work, electrochemical immunosensors have been fabricated for Ricin Chain-A detection. Ricin is one of the most naturally occurring toxins and is produced in the castor oil plant, Ricinus communis. It is a type 2 ribosome-inactivating protein (RIPs) that has two chains (A and B) linked by a disulfide bond. Chain-B facilitates cell binding and entry while chain-A inhibits protein synthesis, causing cell death. For the first time cyclic voltammetry studies have been performed for successful detection of Ricin Chain-A using Ricin singledomain antibodies $\left(\operatorname{Ricin}_{\mathrm{sdAb}}\right)$ based immunosensors. The shelf life of the Ricin ${ }_{\mathrm{sdAb}}$ based immunosensors has also been reported to emphasize the potential sdAbs hold for POC applications. Furthermore, the limitation of the current sensor platform and possible solution is discussed. 


\section{Materials and Methods}

\subsection{Immunosensor fabrication}

Interdigitated microelectrode (IDE) of $10 \mu \mathrm{m}$ width and electrode gap of $10 \mu \mathrm{m}$ was used for fabrication of the immunosensor. IDEs were patterned on 4" oxidized silicon wafers by traditional microfabrication processes (fig. 1a). First, MicroChem $\mathrm{AZ}^{\circledR} 5214$ E photoresist was spin coated on the oxidized wafer, then the IDE pattern was exposed using OAI 800 Mask Aligner. After development in $\mathrm{AZ}^{\circledR} \mathrm{MIF} 300$, chromium (20nm) and gold (200nm) were deposited via electron beam evaporation. The micro IDEs were obtained after lift-off process. Next, MicroChem SU-8 photoresist was patterned to form wells above the IDEs for a fixed volume of $5 \mu \mathrm{L}$.

\subsection{Electrochemical cleaning of IDEs}

The IDEs were electrochemically cleaned prior to bio-immobilization for sensor formation. Electrodes were cleaned through cyclic voltammetry [Autolab Potentiostat / Galvanostat (Eco Chemie, Netherlands)] using $5 \mu \mathrm{L}$ of $0.1 \mathrm{M} \mathrm{H}_{2} \mathrm{SO}_{4}$ as a function of varying scanning cycles at a scan rate of $50 \mathrm{mV} / \mathrm{S}$. The parameters of the IDE cleaning process were optimized using $\mathrm{CV}$ techniques in $5 \mu \mathrm{L}$ PBS (pH 7.4) containing $5 \mathrm{mM} \mathrm{K}\left[\mathrm{Fe}(\mathrm{CN})_{6}\right] /$ $\mathrm{K}_{4}\left[\mathrm{Fe}(\mathrm{CN})_{6}\right]$ at a scan rate of $50 \mathrm{mV} / \mathrm{s}$. The magnitude of current responses obtained was found to increase with increasing cleaning scanning cycles (5-10). Higher current responses were observed in case of IDEs cleaned using 9 cycles and higher. However, the electrode starts to etch after 9 scanning cycles. Thus IDEs were cleaned using 7 scanning cycles in $5 \mu \mathrm{L}, 0.1 \mathrm{M} \mathrm{H}_{2} \mathrm{SO}_{4}$. All IDEs underwent the same cleaning procedure and exhibited identical current response without morphological damage.

\subsection{Immobilization of self-assembled monolayer (SAM) and sdAb onto IDEs}

Ricin $_{\mathrm{sdAb}}$ was obtained from the Center for BioMolecular Science and Engineering, Naval Research Laboratory. Ricin Chain-A antigen were obtained from BEI Resources. Dithiobis (succinimidyl propionate) (DTSP), sodium borohydride $\left(\mathrm{NaBH}_{4}\right)$, and all other chemicals were purchased from Sigma-Aldrich. The chemicals were used as it is, without any further purification. For DTSP based SAM formation, first the $2 \mathrm{mg} / \mathrm{mL}$ solution of DTSP in acetone 
was prepared and reduced using $\mathrm{NaBH}_{4}(10 \mathrm{mg} / \mathrm{mL}$ in DI water). Then the IDEs were immersed in the solution for two hours. After SAM modification, all DTSP/IDEs were rinsed with acetone and then by DI water to remove unbound DTSP particles. Ricin $\operatorname{sdAb}(5 \mu \mathrm{L}, 5.2 \mathrm{mg} / \mathrm{mL})$ was covalently immobilized via a facile reaction between amino group of antibody and reactive succinimidyl group of the DTSP SAM surface. The DTSP-SAM/IDE was incubated with Ricin $_{\text {sdAb }}$ for 2 hours followed by careful washing with IPA and DI water to remove any unbound molecules.

\section{Results and discussion}

\subsection{Structure and stability of single-domain antibody}

Immunosensors commonly rely on traditional monoclonal or polyclonal antibodies derived from mice, rabbits, goats or sheep as recognition elements. These recognition elements perform easily in terms of providing high sensitivity and specificity to the immunosensor. Replacing these conventional antibodies with an immune-reagent that is more stable can simplify the problem of storage and testing under harsher environments. Within the past decade, aptamers (Radi 2011), conventional antibody fragments (Wang and Duan 2011), and single-domain antibodies (Goldman et al. 2006) have gained recognition for their high stability over repeated cycles of denaturation and renaturation as compared to protein based antibodies.

Aptamers i.e. ribonucleic acid (RNA) and single-strand deoxyribonucleic acid (ssDNA) are widely accepted as an alternative class of molecules that can be substituted for in lieu of antibodies (Mascini 2008). They have a defined three-dimensional structure with high affinity and specificity towards their targets. Compared to protein based antibodies, they can recover their original conformation and can bind to targets after being annealed at temperatures up to 40 ${ }^{\circ} \mathrm{C}$ (Jayasena 1999). The synthesis of aptamers is not as laborious as it is for conventional antibodies. They can be reproduced in great quantities with high accuracy via cost effective chemical reactions (Song et al. 2012). One disadvantage is that aptamers for a wide variety of protein targets are still not as widely available as antibodies, which limits their applicability. The search for new aptamers for various targets is still in progress.

Prior to understanding the structure of sdAbs, it is important to understand structure of the conventional antibodies and their fragments. Conventional antibodies are made up of two identical heavy chains and two identical light chains, where the antigen-binding region is formed 
from a variable domain from the heavy chain $(\mathrm{VH})$ paired with a variable domain from the light chain (VL). Recombinant binding elements can be constructed by joining the antibody fragments (VH and VL) with a flexible linker. These recombinant binding elements are referred to as single chain antibodies (scFv) (Nelson 2010). Unfortunately, scFvs are not stable for longer periods of times at temperatures up to $37{ }^{\circ} \mathrm{C}$ (Worn and Pluckthun 2001). Evidently, it is because VH domains are more thermally resistant than VL domains (Wang and Duan 2011). This is, however, not an intrinsic drawback and can be rectified by engineering methodologies. Particular sequences of the $\mathrm{VH}$ domain, $\mathrm{VL}$ domain, and linker chain can be changed to enhance the thermal stability of the scFv structure for longer periods of time at temperatures up to $37{ }^{\circ} \mathrm{C}$ (Willuda et al. 1999; Worn and Pluckthun 2001).

Members of the Camelid family, including camels, llamas and alpaca, possess both conventional antibodies and their unconventional subclasses that consist of only two heavy chains (Hamers-Casterman et al. 1993; Maass et al. 2007). The heavy chain antibody's two antigen binding sites are each formed by only a single variable domain (VHH). These VHHs can be expressed recombinantly and have been termed sdAbs (Arbabi Ghahroudi et al. 1997). They are also referred as nanobodies. Figure $1 \mathrm{~b}$ shows the structure of the conventional antibodies, unconventional antibodies from the camelid family, and their binding fragments. Recent reports suggest that sdAbs are inherently immune to temperature changes (de Marco 2011; Goldman et al. 2008; Goldman et al. 2006; Graef et al. 2011; Liu et al. 2013; Wesolowski et al. 2009). Unlike conventional antibodies, sdAbs have been shown to remain functional at $90{ }^{\circ} \mathrm{C}$ (Goldman et al. 2006; van der Linden et al. 1999). This high temperature stability is mainly attributed to their efficient refolding after chemical or thermal denaturation and, to a lesser extent, an increased resistance against denaturation (Even-Desrumeaux et al. 2010; Saerens et al. 2008). They are also resistant to extreme pH, proteolysis, solvents and detergents (Chames et al. 2009; van der Linden et al. 1999). The ability of these antigen binding molecules to refold after heating to achieve their original structure makes them attractive candidates for diagnostic assays.

Fig. 1(a). Interdigitated electrodes (IDE) for antibody immobilizations to form the biosensor. The process involves (i) fabrication of gold IDEs, (ii) immobilization of DTSP SAM on the Au electrodes. (iii) immobilization of Ricin sdAbs and (iv) electrochemical detection of Ricin Chain-A. 
Fig. 1(b). Structure of (i) conventional antibody with its antigen binding fragment, Single chain antibody ( $\mathrm{scFv}$ ) and (ii) unconventional camelid heavy chain antibody with its antigen binding fragment, single domain antibody (sdAb).

\subsection{Ricin measurement and $\mathrm{pH}$ stability}

The fabricated Ricin $_{\mathrm{sdAb}} / \mathrm{DTSP}-\mathrm{SAM} / \mathrm{IDEs}$ immunoelectrodes (see section 2.3) were used to detect various concentrations of Ricin using CV techniques in $5 \mu \mathrm{L}$ PBS containing $5 \mathrm{mM}$

$\mathrm{K}_{3}\left[\mathrm{Fe}(\mathrm{CN})_{6}\right] / \mathrm{K}_{4}\left[\mathrm{Fe}(\mathrm{CN})_{6}\right]$ (ferri-ferro cyanide) as redox moieties. Ricin $(5 \mu \mathrm{L})$ was placed on the electrochemical immunosensor. The sample was incubated for 30 minutes on the sensor to ensure proper binding. The IDE based sensors were then washed using IPA and DI water to remove unbound antigens. Prior to taking measurements, the influence of $\mathrm{pH}$ was evaluated since the $\mathrm{pH}$ of the supporting electrolyte solution has a profound effect on the electrochemical responses of an immunosensor. In order to evaluate the influence of the $\mathrm{pH}$, the immunosensors were tested with a series of PBS with the $\mathrm{pH}$ ranging from 6.2 to 8 by cyclic voltammetry using ferri-ferro cyanide as redox probe. The experimental results show that an increase in $\mathrm{pH}$ from 6.2 to 7.4 resulted in an increased peak current. Further increment in $\mathrm{pH}$ resulted in the peak current decreasing. These results showed that the maximum current response occurred at pH 7.4 (fig. 2). Therefore, a $\mathrm{pH}$ of 7.4 was maintained for all experiments. The decrease in current response at $\mathrm{pH}$ above 7.4 can be attributed to the decreased biological activity of the antibody in strong alkaline solution .

Fig. 2. Effect of $\mathrm{pH}$ on electrochemical response.

For Ricin measurement, cyclic voltammetry (CV) measurements were performed for Ricin polyclonal antibodies, monoclonal antibodies, and single-domain antibodies. The results from $\mathrm{CV}$ analysis indicated that the magnitude of current response of the redox probe, $\mathrm{Fe}(\mathrm{CN})_{6}{ }^{3-}$ 14- for Ricin ${ }_{s d A b}$ (fig. 3b (iii)) was lower than that of a polyclonal antibody (fig. 3a (i)) and monoclonal antibody (fig. 3a (ii)). This can be explained by the smaller size of the sdAbs. The diminutive size of sdAb ( 12-15kDa) (Eyer and Hruska 2012; Harmsen and De Haard 2007) allows them to bind in spaces inaccessible to conventional monoclonal and polyclonal antibodies. Aptamers ( 5-15kDa) (Lakhin et al. 2013) and scFvs ( $25 \mathrm{kDa})$ (Bird et al. 1988) also have this advantage. Furthermore, the current magnitude of $\mathrm{CV}$ response decreased on addition 
of Ricin antigen (fig. 3b (iv)). This is because the electron transport from electrolyte to IDE surface was inhibited by the formation of insulating immuno-complex between Ricin $_{s d A b}$ and Ricin. The sensor morphology was characterized via electrochemical impedance spectroscopy (EIS) measurements as well. Figure $3 \mathrm{c}$ shows the EIS measurements for each sensor layer: (i) $\mathrm{Au}$ electrode, (ii) SAM layer, (iii) $\operatorname{Ricin}_{\mathrm{sdAb}}$, and (iv) Ricin Chain-A antigen. The measurements were performed in the presence of $\mathrm{Fe}(\mathrm{CN})_{6}{ }^{3-/ 4-}$ redox probe. Since miniaturization is one of the necessities for portability of this POC sensor, CV measurements were performed for further analysis. CV technique is easy to miniaturize and can be integrated with ultra-low-power microelectrics. Previously, a micro-power potentiostat has been demonstrated for POC sensing application by our research group (Cruz et al. 2014).

For further analysis, the current magnitude response for different concentrations $(1 \mathrm{fg} / \mathrm{mL}-$ $1 \mu \mathrm{g} / \mathrm{mL}$ ) of Ricin was measured via $\mathrm{CV}$. The current magnitude was found to decrease with increasing concentration by standard successive addition technique (fig. 4). The detailed successive addition technique can be found in (Arya et al. 2010). Current magnitude vs. logarithm of Ricin concentration follows a linear relation of $y=\left(3 \times 10^{-6}\right)-\left(7 \times 10^{-8}\right) \mathrm{x}$ $\log$ (concentration) from $1 \log (\mathrm{fg} / \mathrm{mL})-1 \log (\mu \mathrm{g} / \mathrm{mL})$ with a detection limit of $1 \log (\mathrm{fg} / \mathrm{mL})$. The $\mathrm{sdAb}$ based immunosensor exhibits a sensitivity of $0.07 \mu \mathrm{A} / \log (\mathrm{g} / \mathrm{mL}) / \mathrm{cm}^{2}$ with a regression coefficient, $R=0.999$. It was observed that the current peak would saturate quickly at 1 $\log (\mu \mathrm{g} / \mathrm{mL})$ or greater, which implies lesser binding sites on sdAbs for Ricin. A limitation on the number of labels or tags that can be incorporated is also imposed by the small size of the singledomain antibody (Goldman et al. 2009) which is not a limitation in this case of tag-free electrochemical sensing. Due to the lesser available binding sites, a lower concentration range was chosen for this experiment. Percentage change in peak current with $1 \log (\mathrm{fg} / \mathrm{mL})$ Ricin from baseline value (only PBS without Ricin) is $36 \%$. From $1 \log (\mathrm{fg} / \mathrm{mL})$ to $1 \log (\mathrm{pg} / \mathrm{mL}), 1$ $\log (\mathrm{pg} / \mathrm{mL})$ to $1 \log (\mathrm{ng} / \mathrm{mL})$ and $1 \log (\mathrm{ng} / \mathrm{mL})$ to $1 \log (\mu \mathrm{g} / \mathrm{mL})$ the percentage change in peak current is $7-10 \%$. For further successive addition the change is much lower and saturates quickly.

Fig. 3(a). The CV response indicates that the ricin (i) polyclonal and (ii) monoclonal antibodies are not stable in a 24 hour period.

Fig. 3(b). CV measurements of three stages of the gold electrode (i): with SAM layer (ii), sdAb (iii), and Ricin Chain-A antigen (iv). The peak's intensity decreases with the addition of each layer. 
Fig. 3(c). EIS measurements of three stages of the gold electrode (i): with SAM layer (ii), sdAb (iii), and Ricin Chain-A antigen (iv). The impedance increases with the addition of each layer.

Fig. 4. Calibration curve obtained from the electrochemical response of the $\operatorname{Ricin}_{\mathrm{sdAb}} / \mathrm{DTSP}$ $\mathrm{SAM} / \mathrm{IDEs}$ immunoelectrode as a function of Ricin Chain-A concentration $(1 \log (\mathrm{fg} / \mathrm{mL})-1$ $\log (\mu \mathrm{g} / \mathrm{mL}))$

\subsection{Thermal stability and shelf-life of sdAb based immunosensors}

To study the thermal stability of the immunosensor, CV measurements were performed after heating the sensor at varied temperatures. Initially thermal stability tests were carried out for SAM on IDEs to take the background response into consideration since SAM stability issues are uniquely defined by the bond between the film and substrate (Srisombat et al. 2011). Short chain $\left(\mathrm{C}_{3}-\mathrm{C}_{5}\right.$; where $\mathrm{C}_{\mathrm{n}}=$ number of $\mathrm{CH}_{2}$ bonds in $\left.\mathrm{CH}_{3}-\mathrm{Cn}-\mathrm{SH}\right)$ thiol based SAM on gold substrate has been reported for desorption at $363 \mathrm{~K}\left(89.95^{\circ} \mathrm{C}\right)$ (Vericat et al. 2010). Therefore, the temperature was varied from $25{ }^{\circ} \mathrm{C}$ to $80{ }^{\circ} \mathrm{C}$ on a single sensor. The sensor was placed on a hot plate and heated for 15 minutes prior to taking CV scans. Since the CV scans were taken in presence of $\mathrm{Fe}(\mathrm{CN})_{6}^{3-/ 4-}$, the sensor was washed with DI water and dried with nitrogen before the next temperature treatment. A shift was observed in the current peak potential from $50{ }^{\circ} \mathrm{C}$, indicating a possible disorientation and blockage resulting in more negative reduction potentials. The lower temperature stability of SAM as compared to the ones reported in literature can be explained by the short 2 hour immersion time for SAM formation. Through scanning tunneling microscopy (STM), the time dependent organization of SAM on gold surfaces has been studied (Li et al. 2006) and the time dependent organization of thiols can be depicted as shown in fig. 5a. A short immersion time of two hours is sufficient for a full coverage but with lesser density and more disorder. Conversely, a densely packed crystalline SAM monolayer is formed during prolonged overnight immersion, or at least 24 hours, as commonly reported in literature. In this research, a shorter SAM formation time of 2 hours was used since the current, due to redox reactions between the electrode and antibody, shows a strong increase in the current with a decrease in the structural integrity (density and disorder or tilt) of the SAM. It should be noted that the current peak from the redox reaction reduces in case of a densely packed crystalline SAM due to charge screening. However, it is easy to fine tune the sensitivity of a sensor with densely packed SAM. 
In the next steps, a CV measurement was carried out for sensors immobilized with $\operatorname{Ricin}_{\mathrm{sdAb}}\left(\operatorname{Ricin}_{\mathrm{SdAb}} / \mathrm{DTSP}\right.$ SAM/IDEs). The temperature was varied from $25{ }^{\circ} \mathrm{C}$ to $50{ }^{\circ} \mathrm{C}$ in increments of $5{ }^{\circ} \mathrm{C}$ on a single sensor. Similar to the steps mentioned in section 3.2 , the sensor was heated, measured, washed, and dried before the next measurement. The data obtained illustrates that the sdAb based electrochemical sensors were stable up to $40{ }^{\circ} \mathrm{C}$ (fig. $6 \mathrm{a}$ ) when compared to Ricin monoclonal and polyclonal antibodies which degrade at room temperature within 24 Hours (fig. 3a). The redox current peaks decreased gradually from $45{ }^{\circ} \mathrm{C}$ onwards and the peak potentials increased. One possible explanation for the increased instability is the gradual increment in disorder of SAM with rising temperature. A disordered SAM tends to incline more towards the electrode surface unlike an ordered SAM which is normal to the electrode surface. An increased disorder in SAM layer implies that there is more coverage of the electrode surface, hence a decrease in the current magnitude. This type of blockage to the sensor surface because of an inclined SAM layer is depicted in fig. 5c. Another possible explanation of the blockage to the sensor surface is the tilt or orientation of the sdAb which is immobilized on the SAM layer. This is depicted by fig $5 \mathrm{~d}$, where the sdAb changes its orientation with rise in temperature and hence blocking electrode surface. A combination of both SAM disorder and $\mathrm{sdAb}$ tilt can also be a possibility as depicted by fig 5e. Based on the aforementioned scenarios, below are the following four cases in sdAb immunosensor:

(1) True positive: No change in redox current peaks for temperatures up to $40{ }^{\circ} \mathrm{C}$

(2) True negative: Reduction in redox current peaks for temperatures greater than $40^{\circ} \mathrm{C}$

(3) False positive: Increment or no reduction in redox current peaks for temperatures greater than $40{ }^{\circ} \mathrm{C}$

(4) False negative: Reduction in redox current peaks for temperatures below $40{ }^{\circ} \mathrm{C}$

Fig. 5. (a) Time dependent organization of SAM monolayer; Depiction of (b) an ordered SAM with sdAb; (c) a disorder SAM with sdAb; (d) an ordered SAM with tilted or disoriented sdAb;

(e) a disordered SAM with tilted sdAb. Possible reasons for increased blockage to the sensor surface with rise in temperature are (c), (d), and (e).

Fig 6 (a). Stability of sdAb at $40{ }^{\circ} \mathrm{C}$ for $24 \mathrm{hrs}$ is confirmed by comparing CV response of (i) $\mathrm{sdAb}$ and (ii) sdAb after $24 \mathrm{hrs}$

Fig. 6(b). A shelf-life of 7 days is confirmed for sdAb by comparison of CV response with (i) sdAb, (ii) Ricin sdAb- antigen at RT, and (iii) ricin sdAb-antigen stored at $40{ }^{\circ} \mathrm{C}$. 
The sdAb immobilized immunosensors which exhibited the true positive nature also demonstrated true negativity, hence the redox current peaks in these sensors reduced for temperatures greater than $40^{\circ} \mathrm{C}$. None of the sensors showed a false positive since the time for SAM formation did not exceed two hours. In the case of a dense SAM layer, the structural integrity of the layer would be maintained and no disorder would be observed. However, blockage to the sensor surface can still result due to the change in sdAb orientation with temperature. Similarly, the false negative nature of the few sensors can be attributed to the poorly formed SAM layer.

For POC and wearable device applications, especially in remote areas, shelf-life of the sensor is crucial for inexpensive methods of export and long term usage. The CV response in fig. $3 \mathrm{a}$ indicates that monoclonal and polyclonal antibodies are not stable at room temperature in a 24 hour period. This limits the application of the electrochemical immunosensors for POC. However, the sdAb immunosensor demonstrated a shelf-life of 1 week (fig. 6b) after being treated to $40^{\circ} \mathrm{C}$ as the maximum temperature. The $\mathrm{CV}$ response of the sdAb immunosensors with Ricin antigen remains the same after 7 days, proving the unaltered condition of the immunosensor during the week. As mentioned earlier, the stability of a sensor is influenced by the SAM stability, antibody stability, and their binding efficiency. Thus, these are the scope for enhancing the sensor's stability as well as shelf-life.

\section{Conclusion}

This paper demonstrates the first prototype of an sdAb based electrochemical immunosensor for POC application. Advantages in terms of thermal stability and shelf-life of the sdAb over the conventional polyclonal and monoclonal antibodies have been reported. The sensor's maximum stability was shown until $40^{\circ} \mathrm{C}$ with a shelf life of seven days. These are highly promising results for using these sensors in POC and wearable devices cost effectively. A short two hour duration

for SAM formation was employed for maximum current response. However, this limited the packing density of the SAM monolayer on the electrode and thereby its stability. With this tradeoff under consideration further studies are in progress to enhance the sensor's thermal stability with longer immersion time while maintaining maximum current response.

\section{Acknowledgements}


The authors would like to acknowledge Dr. Ellen Goldman (Research Biologist at Naval Research Laboratory (NRL)) for her guidance and the Center for BioMolecular Science and Engineering, NRL for providing the single-domain antibodies without which this work would not have been possible. Authors would also like to acknowledge the Advanced Materials Engineering Research Institute (AMERI), at Florida International University for research facilities. This project is being funded by the NSF Nanosystems Engineering Research Centre for Advanced Self-Powered Systems of Integrated Sensors and Technologies (Award Number: 1160483). Thanks to Dr. Ajeet Kaushik, Assistant Professor at Herbert Wertheim College of Medicine, FIU, for the healthy discussions and suggestions.

\section{References}

Introduction to antibodies. 2nd ed. Chemicon International Inc. 1998.

Protein stability and storage. p. 3. Pierce Biotechnology Inc. 2005.

Anderson, G.P., Bernstein, R.D., Swain, M.D., Zabetakis, D., Goldman, E.R., 2010. Binding Kinetics of Antiricin Single Domain Antibodies and Improved Detection Using a B Chain Specific Binder. Analytical Chemistry 82(17), 7202-7207.

Anderson, G.P., Liu, J.L., Hale, M.L., Bernstein, R.D., Moore, M., Swain, M.D., Goldman, E.R., 2008. Development of Antiricin Single Domain Antibodies Toward Detection and Therapeutic Reagents. Analytical Chemistry 80(24), 9604-9611.

Arbabi Ghahroudi, M., Desmyter, A., Wyns, L., Hamers, R., Muyldermans, S., 1997. Selection and identification of single domain antibody fragments from camel heavy-chain antibodies. FEBS Letters 414(3), 521-526.

Arya, S.K., Chornokur, G., Venugopal, M., Bhansali, S., 2010. Dithiobis(succinimidyl propionate) modified gold microarray electrode based electrochemical immunosensor for ultrasensitive detection of cortisol. Biosensors and Bioelectronics 25(10), 2296-2301.

Bird, R.E., Hardman, K.D., Jacobson, J.W., Johnson, S., Kaufman, B.M., Lee, S.M., Lee, T., Pope, S.H., Riordan, G.S., Whitlow, M., 1988. Single-chain antigen-binding proteins. Science 242(4877), 423-426.

Brinker, C.J., Ashley, C.S., Bhatia, R., Singh, A.K., 2002. Sol-gel method for encapsulating molecules. Google Patents.

Campuzano, S., Salema, V., Moreno-Guzmán, M., Gamella, M., Yáñez-Sedeño, P., Fernández, L.A., Pingarrón, J.M., 2014. Disposable amperometric magnetoimmunosensors using nanobodies as biorecognition element. Determination of fibrinogen in plasma. Biosensors and Bioelectronics 52, 255260.

Chames, P., Van Regenmortel, M., Weiss, E., Baty, D., 2009. Therapeutic antibodies: successes, limitations and hopes for the future. British Journal of Pharmacology 157(2), 220-233.

Cruz, A.F.D., Norena, N., Kaushik, A., Bhansali, S., 2014. A low-cost miniaturized potentiostat for pointof-care diagnosis. Biosensors and Bioelectronics 62, 249-254.

de Marco, A., 2011. Biotechnological applications of recombinant single-domain antibody fragments. Microbial Cell Factories 10, 44-44.

Even-Desrumeaux, K., Baty, D., Chames, P., 2010. Strong and oriented immobilization of single domain antibodies from crude bacterial lysates for high-throughput compatible cost-effective antibody array generation. Molecular Biosystems 6(11), 2241-2248. 
Eyer, L., Hruska, K., 2012. Single-domain antibody fragments derived from heavy-chain antibodies: a review. Veterinary Medicine 57(9), 439-513.

Goldman, E., Liu, J., Bernstein, R., Swain, M., Mitchell, S., Anderson, G., 2009. Ricin Detection Using Phage Displayed Single Domain Antibodies. Sensors 9(1), 542-555.

Goldman, E.R., Anderson, G.P., Conway, J., Sherwood, L.J., Fech, M., Vo, B., Liu, J.L., Hayhurst, A., 2008. Thermostable Llama Single Domain Antibodies for Detection of Botulinum A Neurotoxin Complex. Analytical Chemistry 80(22), 8583-8591.

Goldman, E.R., Anderson, G.P., Liu, J.L., Delehanty, J.B., Sherwood, L.J., Osborn, L.E., Cummins, L.B., Hayhurst, A., 2006. Facile Generation of Heat Stable Antiviral and Antitoxin Single Domain Antibodies from a Semi-synthetic Llama Library. Analytical Chemistry 78(24), 8245-8255.

Graef, R.R., Anderson, G.P., Doyle, K.A., Zabetakis, D., Sutton, F.N., Liu, J.L., Serrano-González, J., Goldman, E.R., Cooper, L.A., 2011. Isolation of a highly thermal stable lama single domain antibody specific for Staphylococcus aureus enterotoxin B. BMC biotechnology 11(1), 86.

Hamers-Casterman, C., Atarhouch, T., Muyldermans, S., Robinson, G., Hammers, C., Bajyana Songa, E., Bendahman, N., Hammers, R., 1993. Naturally occurring antibodies devoid of light chains. Nature 363, 446-448.

Harmsen, M.M., De Haard, H.J., 2007. Properties, production, and applications of camelid single-domain antibody fragments. Applied Microbiology and Biotechnology 77(1), 13-22.

Huang, L., Reekmans, G., Saerens, D., Friedt, J.-M., Frederix, F., Francis, L., Muyldermans, S., Campitelli, A., Hoof, C.V., 2005. Prostate-specific antigen immunosensing based on mixed self-assembled monolayers, camel antibodies and colloidal gold enhanced sandwich assays. Biosensors and Bioelectronics 21(3), 483-490.

Jayasena, S.D., 1999. Aptamers: an emerging class of molecules that rival antibodies in diagnostics. Clinical Chemistry 45(9), 1628-1650.

Kaushik, A., Arya, S.K., Vasudev, A., Bhansali, S., 2013. Nanocomposites based on chitosan-metal/metal oxides hybrids for biosensors applications. Journal of Nanoscience Letters 3(32), 18.

Lakhin, A.V., Tarantul, V.Z., Gening, L.V., 2013. Aptamers: Problems, Solutions and Prospects. Acta Naturae 5(4), 34-43.

Lan, E.H., Dunn, B., Zink, J.I., 2000. Sol-Gel Encapsulated Anti-Trinitrotoluene Antibodies in Immunoassays for TNT. Chemistry of Materials 12(7), 1874-1878.

Li, S.-S., Xu, L.-P., Wan, L.-J., Wang, S.-T., Jiang, L., 2006. Time-Dependent Organization and Wettability of Decanethiol Self-Assembled Monolayer on Au(111) Investigated with STM. The Journal of Physical Chemistry B 110(4), 1794-1799.

Lillehoj, P.B., Huang, M.-C., Truong, N., Ho, C.-M., 2013. Rapid electrochemical detection on a mobile phone. Lab on a Chip 13(15), 2950-2955.

Liu, J.L., Zabetakis, D., Lee, A.B., Goldman, E.R., Anderson, G.P., 2013. Single domain antibody-alkaline phosphatase fusion proteins for antigen detection - Analysis of affinity and thermal stability of single domain antibody. Journal of Immunological Methods 393(1-2), 1-7.

Loncaric, C., Tang, Y., Ho, C., Parameswaran, M.A., Yu, H.-Z., 2012. A USB-based electrochemical biosensor prototype for point-of-care diagnosis. Sensors and Actuators B: Chemical 161(1), 908-913.

Maass, D.R., Sepulveda, J., Pernthaner, A., Shoemaker, C.B., 2007. Alpaca (Lama pacos) as a convenient source of recombinant camelid heavy chain antibodies (VHHs). Journal of Immunological Methods 324(1-2), 13-25.

MacCraith, B.D., McDonagh, C.M., O'Keeffe, G., McEvoy, A.K., Butler, T., Sheridan, F.R., 1995. Sol-gel coatings for optical chemical sensors and biosensors. Sensors and Actuators B: Chemical 29(1-3), 51-57. Mascini, M., 2008. Aptamers and their applications. Anal Bioanal Chem 390(4), 987-988.

Nelson, A.L., 2010. Antibody fragments: Hope and hype. Monoclonal Antibodies (mAbs) 2(1), 77-83. 
Radi, A.-E., 2011. Electrochemical Aptamer-Based Biosensors: Recent Advances and Perspectives. International Journal of Electrochemistry 2011, 17.

Saerens, D., Huang, L., Bonroy, K., Muyldermans, S., 2008. Antibody Fragments as Probe in Biosensor Development. Sensors 8(8), 4669.

Slocik, J.M., Kim, S.N., Auvil, T., Goldman, E.R., Liu, J., Naik, R.R., 2010. Single domain antibody templated nanoparticle resistors for sensing. Biosensors and Bioelectronics 25(8), 1908-1913.

Song, K.-M., Lee, S., Ban, C., 2012. Aptamers and Their Biological Applications. Sensors (Basel, Switzerland) 12(1), 612-631.

Srisombat, L., Jamison, A.C., Lee, T.R., 2011. Stability: A key issue for self-assembled monolayers on gold as thin-film coatings and nanoparticle protectants. Colloids and Surfaces A: Physicochemical and Engineering Aspects 390(1-3), 1-19.

Swed, A., Cordonnier, T., Fleury, F., Boury, F., 2014. Protein Encapsulation into PLGA Nanoparticles by a Novel Phase Separation Method Using Non-Toxic Solvents. Journal of Nanomedicine and Nanotechnolgy $5,241$.

van der Linden, R.H.J., Frenken, L.G.J., de Geus, B., Harmsen, M.M., Ruuls, R.C., Stok, W., de Ron, L., Wilson, S., Davis, P., Verrips, C.T., 1999. Comparison of physical chemical properties of llama VHH antibody fragments and mouse monoclonal antibodies. Biochimica et Biophysica Acta (BBA) - Protein Structure and Molecular Enzymology 1431(1), 37-46.

Vericat, C., Vela, M.E., Benitez, G., Carro, P., Salvarezza, R.C., 2010. Self-assembled monolayers of thiols and dithiols on gold: new challenges for a well-known system. Chemical Society Reviews 39(5), 18051834.

Wan, Y., Su, Y., Zhu, X., Liu, G., Fan, C., 2013. Development of electrochemical immunosensors towards point of care diagnostics. Biosensors and Bioelectronics 47(0), 1-11.

Wang, T., Duan, Y., 2011. Probing the stability-limiting regions of an antibody single-chain variable fragment: a molecular dynamics simulation study. Protein Engineering, Design and Selection 24(9), 649657.

Wesolowski, J., Alzogaray, V., Reyelt, J., Unger, M., Juarez, K., Urrutia, M., Cauerhff, A., Danquah, W., Rissiek, B., Scheuplein, F., Schwarz, N., Adriouch, S., Boyer, O., Seman, M., Licea, A., Serreze, D.V., Goldbaum, F.A., Haag, F., Koch-Nolte, F., 2009. Single domain antibodies: promising experimental and therapeutic tools in infection and immunity. Medical Microbiology and Immunology 198(3), 157-174.

Willuda, J., Honegger, A., Waibel, R., Schubiger, P.A., Stahel, R., Zangemeister-Wittke, U., Pluckthun, A., 1999. High thermal stability is essential for tumor targeting of antibody fragments: engineering of a humanized anti-epithelial glycoprotein-2 (epithelial cell adhesion molecule) single-chain Fv fragment. Cancer Research 59(22), 5758-5767.

Worn, A., Pluckthun, A., 2001. Stability engineering of antibody single-chain Fv fragments. Journal of Molecular Biology 305(5), 989-1010.

Zhu, Z., Shi, L., Feng, H., Susan Zhou, H., 2015. Single domain antibody coated gold nanoparticles as enhancer for Clostridium difficile toxin detection by electrochemical impedance immunosensors. Bioelectrochemistry 101, 153-158. 


\section{List of figures}

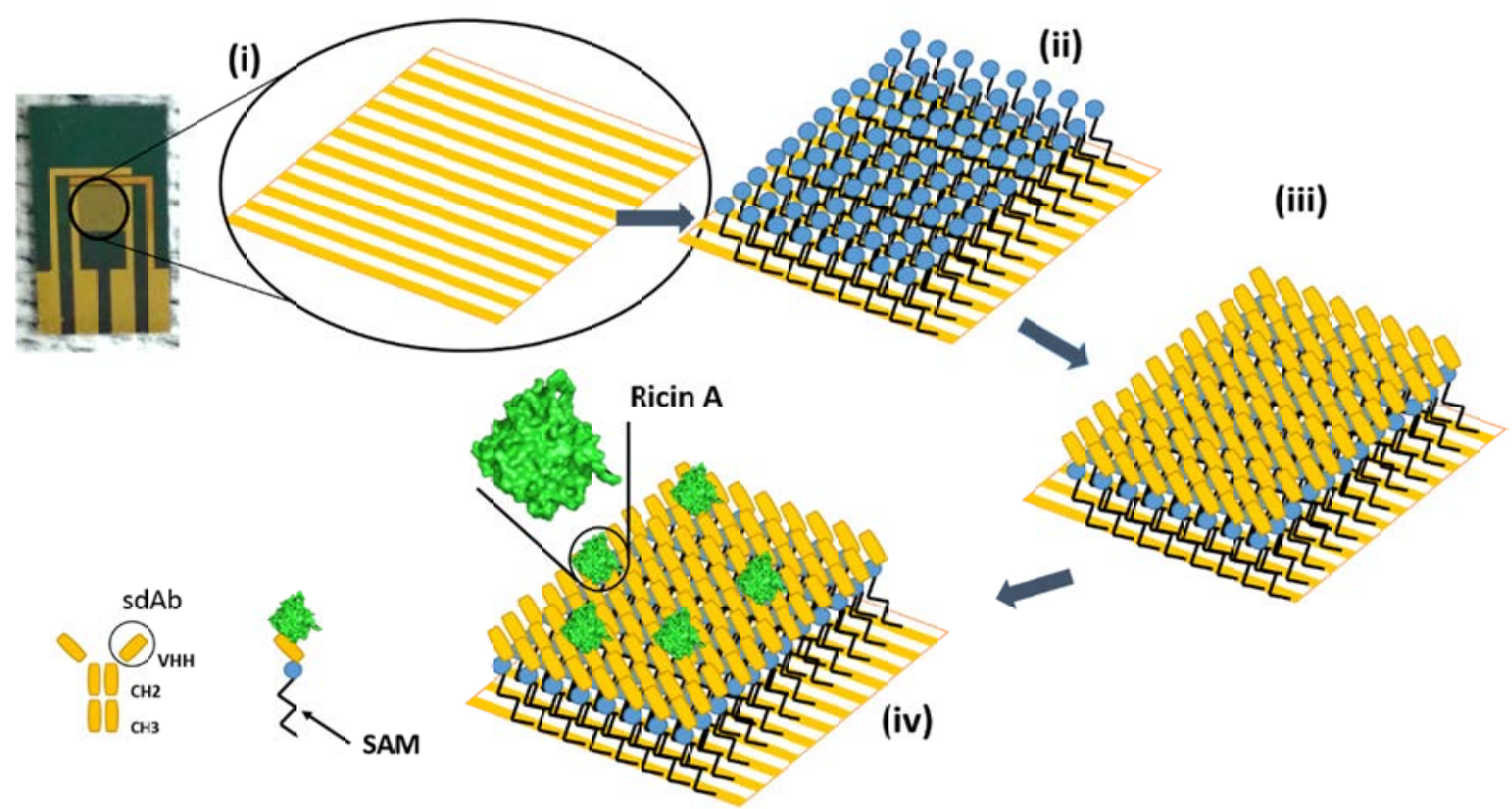

Fig. 1(a). Interdigitated electrodes (IDE) for antibody immobilizations to form the biosensor. The process involves (i) fabrication of gold IDEs, (ii) immobilization of DTSP SAM on the Au electrodes. (iii) immobilization of Ricin sdAbs and (iv) electrochemical detection of Ricin

Chain-A.

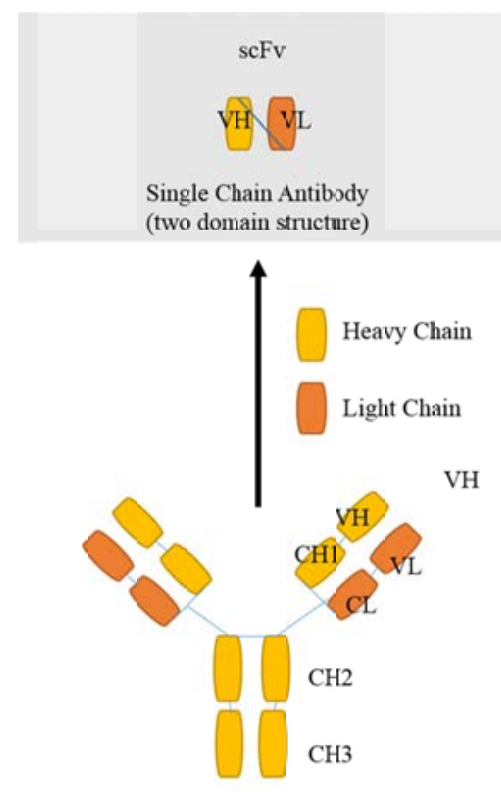

(i)

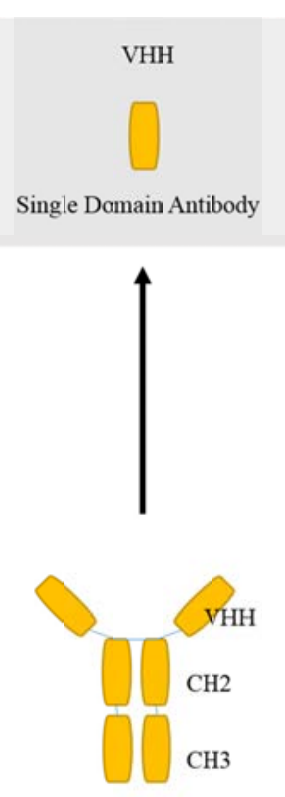

(ii)

Fig. 1(b). Structure of (i) conventional antibody with its antigen binding fragment, Single chain antibody (scFv) and (ii) unconventional camelid heavy chain antibody with its antigen binding fragment, single domain antibody (sdAb) 


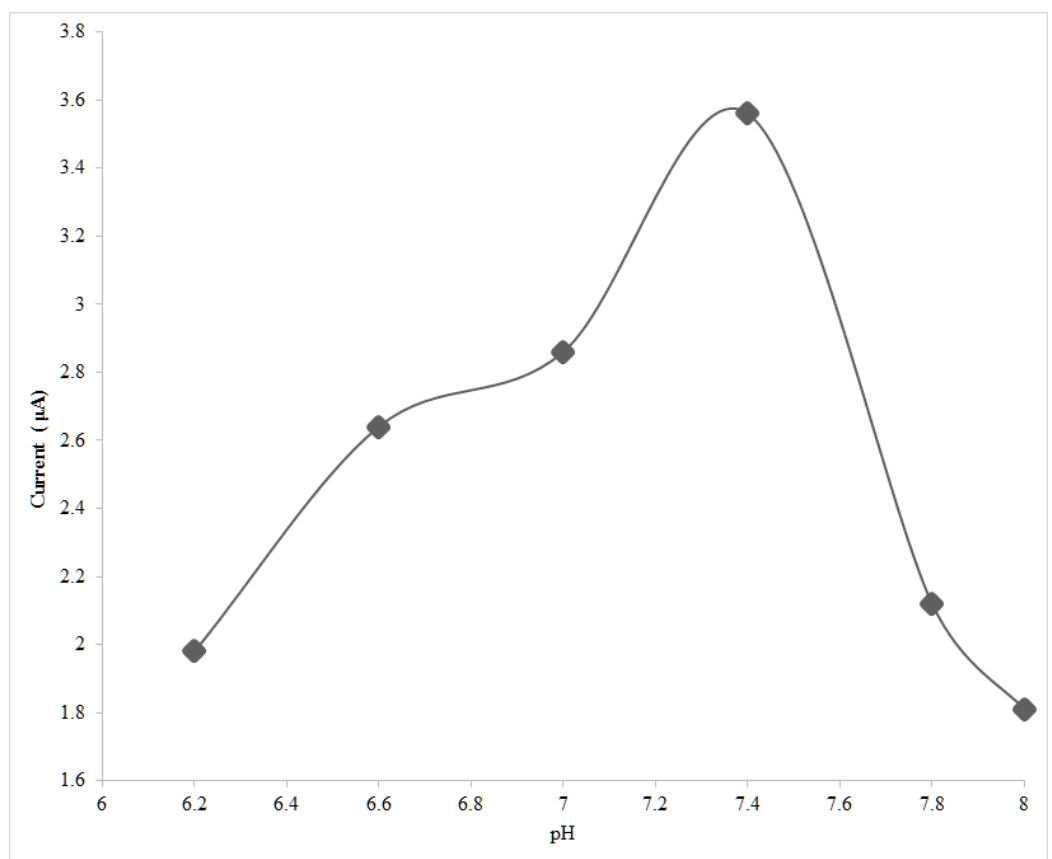

Fig. 2. Effect of $\mathrm{pH}$ on electrochemical response.
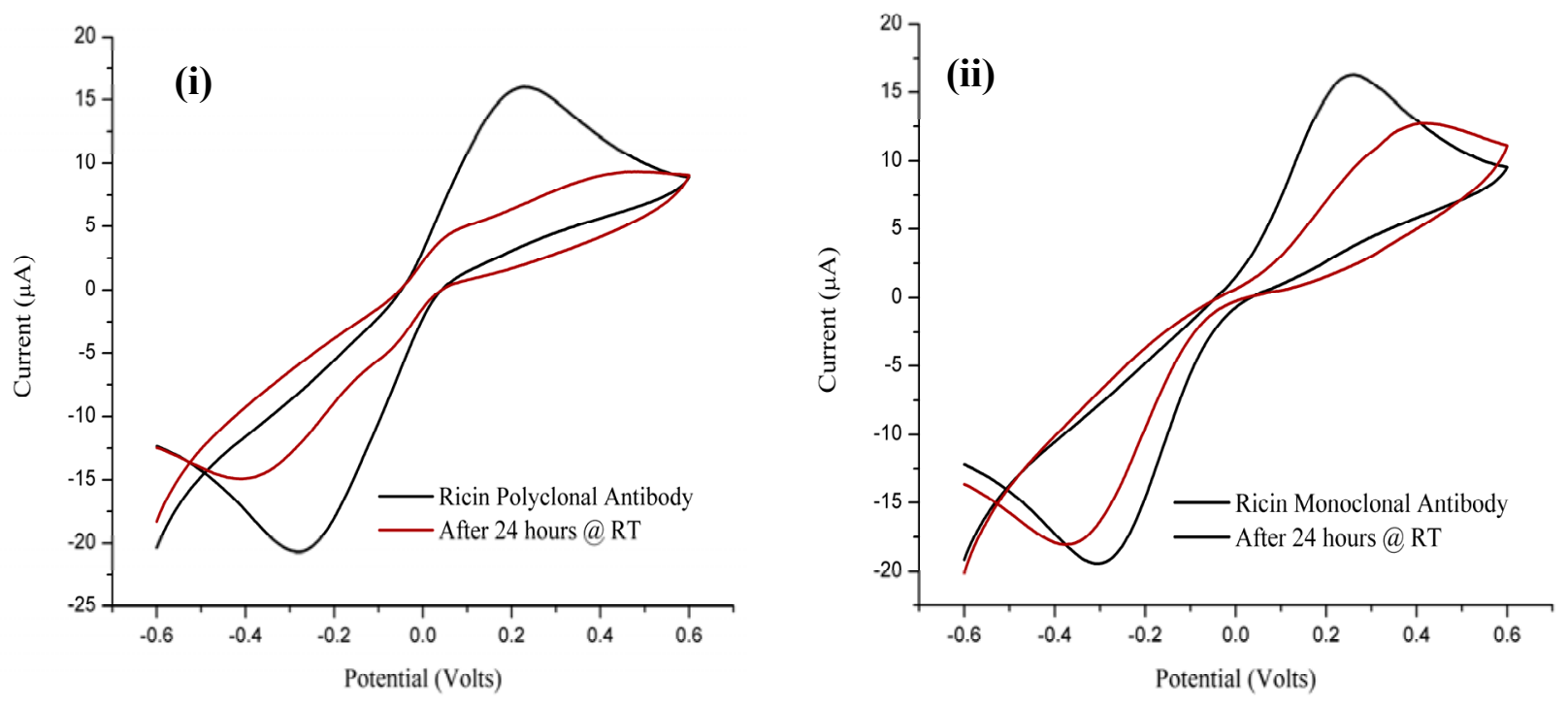

Fig. 3(a). The CV response indicates that the ricin (i) polyclonal and (ii) monoclonal antibodies are not stable in a 24 hour period. 


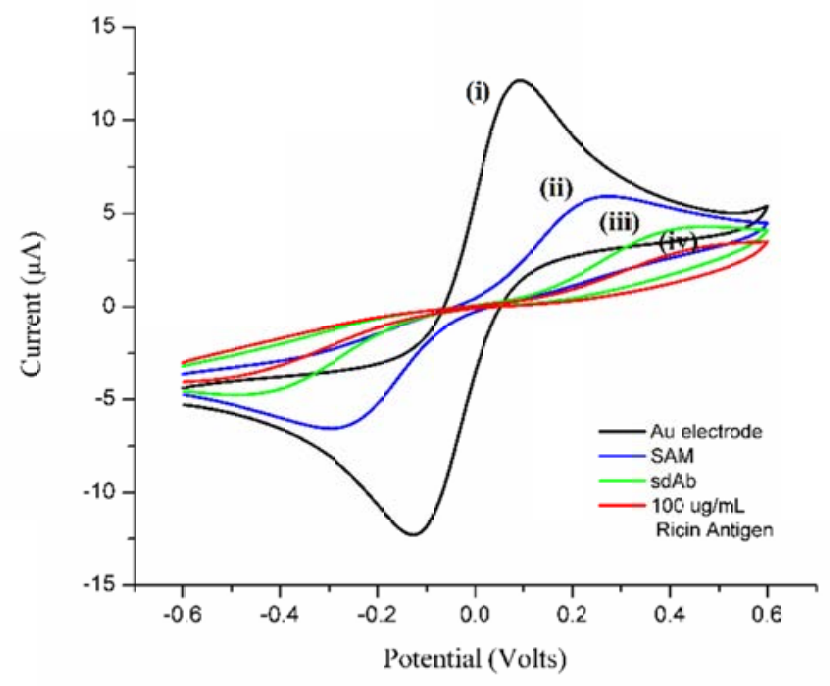

Fig. 3(b). CV measurements of three stages of the gold electrode (i): with SAM layer (ii), sdAb (iii), and Ricin Chain-A antigen (iv). The peak's intensity decreases with the addition of each layer.

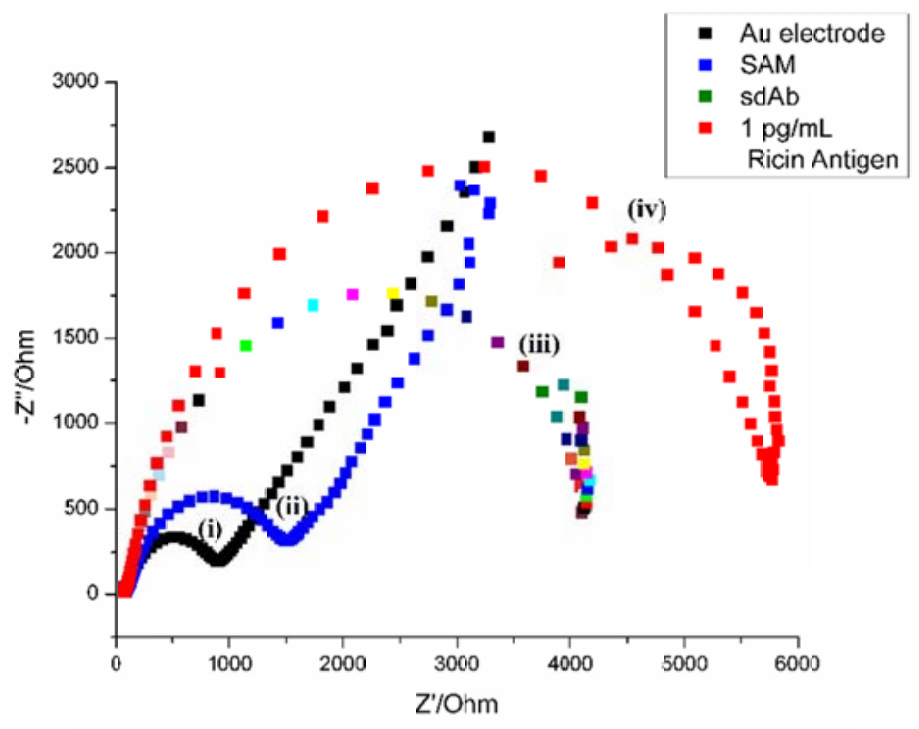

Fig. 3(c). EIS measurements of three stages of the gold electrode (i): with SAM layer (ii), sdAb (iii), and Ricin Chain-A antigen (iv). The impedance increases with the addition of each layer. 


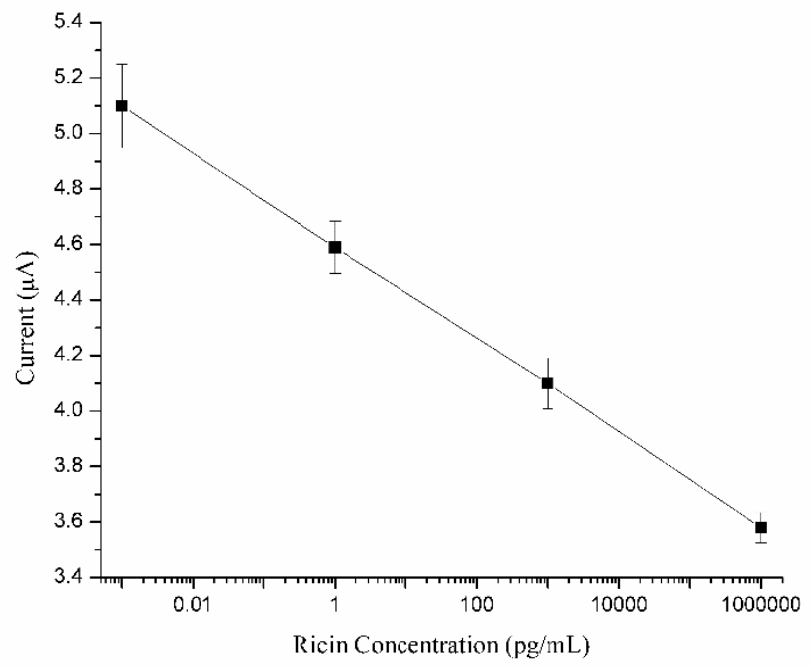

Fig. 4. Calibration curve obtained from the electrochemical response of the $\operatorname{Ricin}_{\mathrm{sdAb}} / \mathrm{DTSP}-$ SAM/IDEs immunoelectrode as a function of Ricin Chain-A concentration $(1 \log (\mathrm{fg} / \mathrm{mL})-1$ $\log (\mu \mathrm{g} / \mathrm{mL}))$

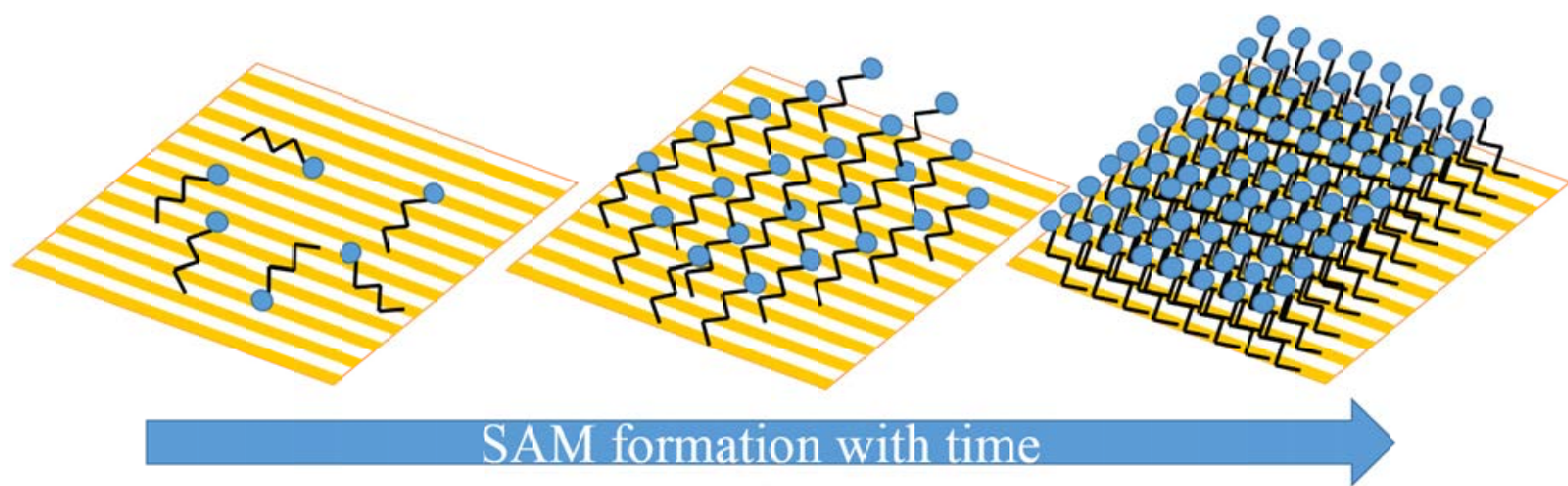

(a)

(b)

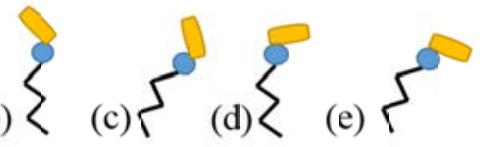

Fig. 5. (a) Time dependent organization of SAM monolayer; Depiction of (b) an ordered SAM with $\mathrm{sdAb}$; (c) a disorder SAM with sdAb; (d) an ordered SAM with tilted or disoriented sdAb; (e) a disordered SAM with tilted sdAb. Possible reasons for increased blockage to the sensor surface with rise in temperature are (c), (d), and (e). 


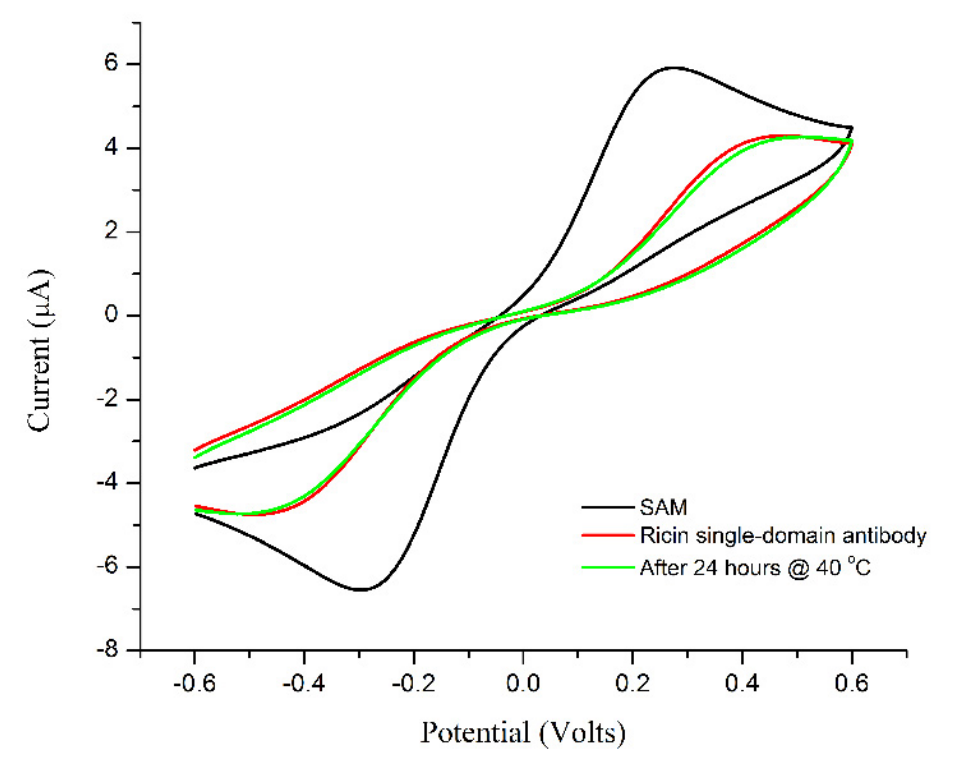

Fig 6 (a). Stability of sdAb at $40{ }^{\circ} \mathrm{C}$ for 24 hrs is confirmed by comparing CV response of (i) $\mathrm{sdAb}$ and (ii) sdAb after $24 \mathrm{hrs}$

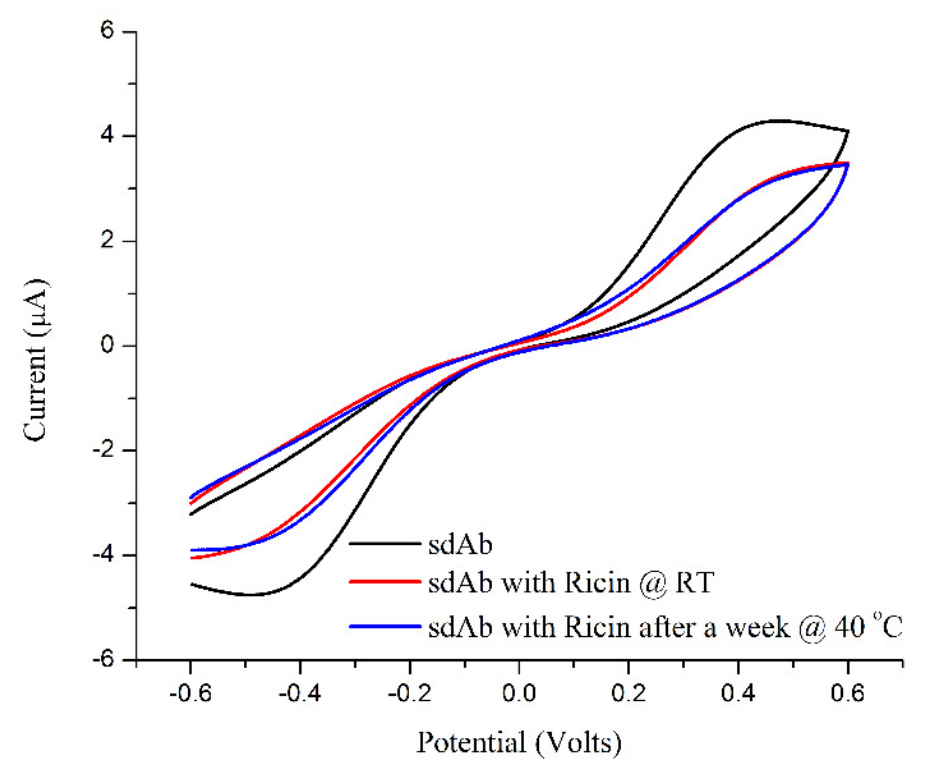

Fig. 6(b). A shelf-life of 7 days is confirmed for sdAb by comparison of CV response with (i) sdAb, (ii) Ricin sdAb- antigen at RT, and (iii) ricin sdAb-antigen stored at $40{ }^{\circ} \mathrm{C}$. 